\title{
PREVALENCE OF RUBELLA VIRUS-SPECIFIC IMMUNOGLOBULIN-G AND -M IN PREGNANT WOMEN ATTENDING TWO TERTIARY HOSPITALS IN SOUTHWESTERN NIGERIA
}

\author{
OBIJIMI, ${ }^{1}$ T. O., AJETOMOBI ${ }^{1}$, A. B., SULE, ${ }^{1}$ W. F. \& OLUWAYELU, ${ }^{2}$ D. O. \\ ${ }^{1}$ Department of Biological Sciences, Osun State University, PMB 4494, Osogbo, Osun State, Nigeria; ${ }^{2}$ Department of Veterinary \\ Microbiology \& Parasitology, Faculty of Veterinary Medicine, University of Ibadan, Oyo State, Nigeria. \\ Correspondence: SULE Waidi Folorunso, Department of Biological Sciences, Osun State University, PMB 4494, Osogbo, Osun \\ State. 08032071447; equine318@yahoo.com
}

\begin{abstract}
Background: Rubella is a self-limiting disease that causes congenital rubella syndrome (CRS) when rubella virus (RV) infects women in the first trimester of pregnancy.

Objective: To assess a population of pregnant women attending antenatal clinics in two tertiary hospitals in southwestern Nigeria for anti-RV IgG and IgM in order to determine the proportions susceptible to the viral infection and infectious to their fetuses.

Method: Ninety consenting pregnant women were consecutively recruited and aseptically bled for the study. The sera were screened with commercial ELISA kits for anti-rubella virus IgG and IgM. Due to the controls and calibrators included in the tests, the IgG test was performed on 89 sera while all 90 samples were screened for IgM.

Results: Of the 89 pregnant women tested for anti-RV IgG, $86(96.6 \%)$ were positive with protective serum concentrations of the antibody. Only six $(6.7 \%, 95 \%$ CI: $1.5-11.8 \%, n=90)$ women were positive for anti-RV IgM with $5(5.6 \%, 95 \%$ CI: $0.8-10.3 \%)$ having both anti-RV IgG and IgM. Overall, three (3.4\%) of the women were susceptible to RV infection, one (1.1\%) of these in the first trimester of gestation was probably infectious.

Conclusion: Majority of the pregnant women had protective levels of anti-RV IgG antibody although susceptibility to rubella as well as level of infectiousness were low. Intensification of rubella immunization of all females of child-bearing age in Osun and Oyo States is advocated.
\end{abstract}

Keywords: Prevalence, Rubella, Antibodies, Pregnant women, Southwestern Nigeria.

\section{INTRODUCTION}

Rubella, a vaccine-preventable viral disease, is predominantly a human disease that occurs between infancy and puberty (1). As a mild exanthematous disease, it is associated with low-grade fever, lymphadenopathy, headache, malaise, mild coryza, and conjunctivitis with a short-lived (acute) maculopapular rash $(2,3,4)$. The etiologic agent rubella virus (RV) - is an enveloped, positive-sense, ssRNA virus in the genus Rubivirus of the family Togaviridae. Individuals infected with RV are most infectious when the rash is erupting, but they may shed virus from 7 days before to 14 days after the onset of rash. Following exposure, the incubation period before onset of symptoms is usually 14-18 days (range 12-23 days) (5).

Prior to the introduction of immunization programs, rubella was endemic worldwide and peak infection occurred in the 5-9 year-old age group. After vaccine implementation, the disease shifted from children to young adults until its virtual elimination from North America and Europe in recent years $(6,7)$. In tropical countries however, epidemics still occur but the lack of effective monitoring programs coupled with the absence of serious clinical symptoms in affected children, make the epidemics difficult to assess (8). In countries that have not implemented vaccination programs, infection at an early age is still the norm, with high seroconversion rates found in both preschool populations and in the 5 to 9 year-old age group (9). However, a substantial number of women of childbearing age (10-25\%) were reportedly susceptible to rubella (10).

Cell-mediated and humoral immunity develop in humans following natural RV infection and with rubella immunization (11). Antibodies produced against RV comprise the primary immunoglobulin M (IgM) and later IgG which are the markers of current RV infection and past infection or protective immunity respectively. With natural infection, RVspecific IgM antibodies become detectable within 3-4 days and IgG antibodies within one week of the onset of rash while following rubella vaccination, the appearance of RV-specific IgM and IgG antibodies is somewhat delayed and peak levels are lower compared to natural infection. Rubella-specific IgM can often be detected in individuals up to two months after illness and in a decreasing percentage of 
individuals up to six to seven months after natural infection, vaccination and re-infection (11). Vaccination of children and mothers with RV vaccine is capable of conferring herd immunity that interrupts RV transmission. Vaccinated or naturally-infected mothers with subsequent seroconversion pass protective anti-RV IgG to their newborns $(3,12)$. It is noteworthy however that false-positive $\operatorname{IgM}$ test results may occur due to cross-reacting IgM antibodies to Epstein Barr virus, human parvovirus B19, rheumatoid factor or other auto-antibodies.

Though males and females are susceptible, RV infection becomes more important when the fetuses of pregnant women are infected during the first trimester of pregnancy. This usually results in serious consequences such as miscarriages, stillbirths and a constellation of severe birth defects known as congenital rubella syndrome (CRS). Ninety per cent $(90 \%)$ of mothers infected during the first 11 or 12 weeks of gestation will deliver an infant with CRS and the most common congenital defects of CRS are cataracts, heart defects and hearing impairment, mental/physical retardation et cetera $(4,13)$. The primary objective of the rubella immunization program therefore is to prevent CRS.

Immunity to RV is most frequently ascertained by detection of specific antibodies using enzyme-linked immunosorbent assay (ELISA) or other immunoassay methods $(5,14,15)$. In Nigeria, Odelola et al. (16) in a multicenter study involving Northern, Eastern and Western Nigeria, reported that an average of $68 \%$ of the Nigerian population possessed rubella virus antibodies while Pennap et al. (17) reported a 3.9\% prevalence of RV IgM among pregnant women in Makurdi, Benue State. Other studies among pregnant women detected rubella IgG antibodies of $68.5 \%$ in Ibadan (18), 54.1\% in Maiduguri (19) and 76\% in Lagos (20). The present study was conducted to determine the proportion of pregnant women with anti-rubella virus $\operatorname{IgG}$ and $\operatorname{IgM}$ in two tertiary hospitals in southwestern Nigeria; to assess the rate of susceptibility to rubella virus infection and determine the possibility of vertical transmission of the virus.

\section{MATERIALS AND METHODS}

\section{Study area and population}

This study was carried out in Wesley Guild Hospital, Ilesa, Osun State and Jericho Nursing Home, Ibadan, Oyo State between October, 2011 and May, 2012. Wesley Guild Hospital is located in Ilesa West local government area (LGA) of Osun State while Jericho Nursing Home is in Ido LGA, Oyo State. Figure 1 shows the demographics of the women.

\section{Study design}

For this study, a cross-sectional, hospital-based design was employed following ethical approval by the Health Research Ethical Committee of the College of Health Sciences, Osun State University, Osogbo. A designated physician (Obstetrician) in the two hospitals discussed the objectives and procedures of this study with pregnant women visiting the antenatal clinic of both hospitals. Ninety pregnant women that consented to participate in the study were consecutively recruited. Each pregnant woman provided pertinent demographic data that were obtained through interviewer-administered questionnaires. These data included age, report of MMR vaccination, educational status, marital status, trimester of pregnancy, number of pregnancy (parity), and present/past experience of skin rash, history of family member with skin rash and knowledge of rubella.

\section{Blood sample collection/serum preparation}

About $5 \mathrm{ml}$ blood sample was aseptically collected by venipuncture from each pregnant woman. Each blood sample was dispensed into appropriately labeled anticoagulant-free sample tubes, screw-capped and left at room temperature for about 45 minutes to 1 hour, after which it was spun at 3,000 revolutions per minute for 10 minutes to separate serum from blood clot. The serum was dispensed into correspondingly labeled Eppendorf tube and stored at $-20^{\circ} \mathrm{C}$ until tested.

\section{Serological analysis of samples}

The sera of the pregnant women were tested for antiRV IgG and IgM using commercial ELISA kits - RUB IgG ELISA for the quantitative/qualitative determination of IgG antibodies and RUB IgM "Capture" for the determination of IgM antibodies to rubella virus in human serum and plasma (DIA.PRO, Diagnostic Bioprobes Srl, Sesto San Giovanni, Milano, Italy). The serologic tests and interpretation of results were done in accordance with the manufacturer's instructions while optical signals generated were read at $450 \mathrm{~nm}$ with ELISA plate reader (Optic Ivymen ${ }^{\circledR}$ System, Model 2100C). Due to the controls and calibrators included in the tests, the IgG test was performed on 89 sera while all 90 samples were screened for IgM.

\section{Interpretation of ELISA results}

According to the IgG ELISA kit protocol, serum samples with anti-RV IgG concentrations < $10 \mathrm{WHO}$ $\mathrm{IU} / \mathrm{ml}$ were considered negative for anti-RV IgG antibody while those with concentrations $\geq 10 \mathrm{WHO}$ $\mathrm{IU} / \mathrm{ml}$ were considered positive. The latter titer is considered the lowest concentration of IgG that provides an effective immunological protection against a second infection of RV. Therefore, for the purpose of determining the seropositivity and 
corresponding concentration of anti-RV IgG in each serum sample, the lower limit of the serum control (i.e. $18 \mathrm{IU} / \mathrm{ml}$ of anti-rubella virus IgG equivalent to OD of 0.75) was used to estimate the IgG concentration. For instance, serum sample 1 recorded OD of 1.304 which is equivalent to $31.296 \mathrm{IU} / \mathrm{ml}$ of anti-RV IgG. The pregnant woman having this sample was hence considered seropositive with protective level of anti-RV IgG. This estimation was done for each of the 89 serum samples.

For the IgM ELISA, serum samples with Sample to Cut-off (S/Co) ratio > 1.2 were considered positive for anti-RV IgM antibodies while those with S/Co ratio < 1.0 were considered negative. Samples with S/Co ratio between 1.0 and 1.2 were considered equivocal as recommended by the kit manufacturer.

\section{Data analysis}

The results obtained were analyzed using descriptive statistics i.e. mean and percentages with $95 \%$ confidence interval (CI) but in order to prevent report of negative values, CI was not estimated for proportions close to $0.0 \%$ and $100.0 \%$. For statistical comparison of the mean age and educational status of the women from Ilesa and Ibadan, independent t-test and Chi-square test were used while to establish significant differences between groups, we used independent t-test (for two groups) and ANOVA (for more than 2 groups) for average values of serum concentration of anti-RV IgG. For IgM seropositivity, Chi-square test was used and p-values $<0.05$ served as indicator of statistical significance. The data analysis was done with SPSS version 15.0 for Windows (SPSS Inc., Chicago, IL).

\section{RESULTS}

The overall age of the study participants ranged from 19-44 years (yrs) (mean age: 30.8 [95\% CI: 29.9-31.7] yrs). The demographic characteristics of the pregnant women involved in the study are shown in Figure 1. It was observed that the pregnant women from Ilesa were statistically comparable in age $(P=0.31)$ and educational status $(\mathrm{P}=0.18)$ with those from Ibadan.

Five pregnant women (3 from Ilesa and 2 from Ibadan) were positive for both anti-RV IgG and IgM antibodies giving an overall prevalence rate of $5.6 \%$ (95\% CI: 0.8-10.3, $\mathrm{n}=90$ ).

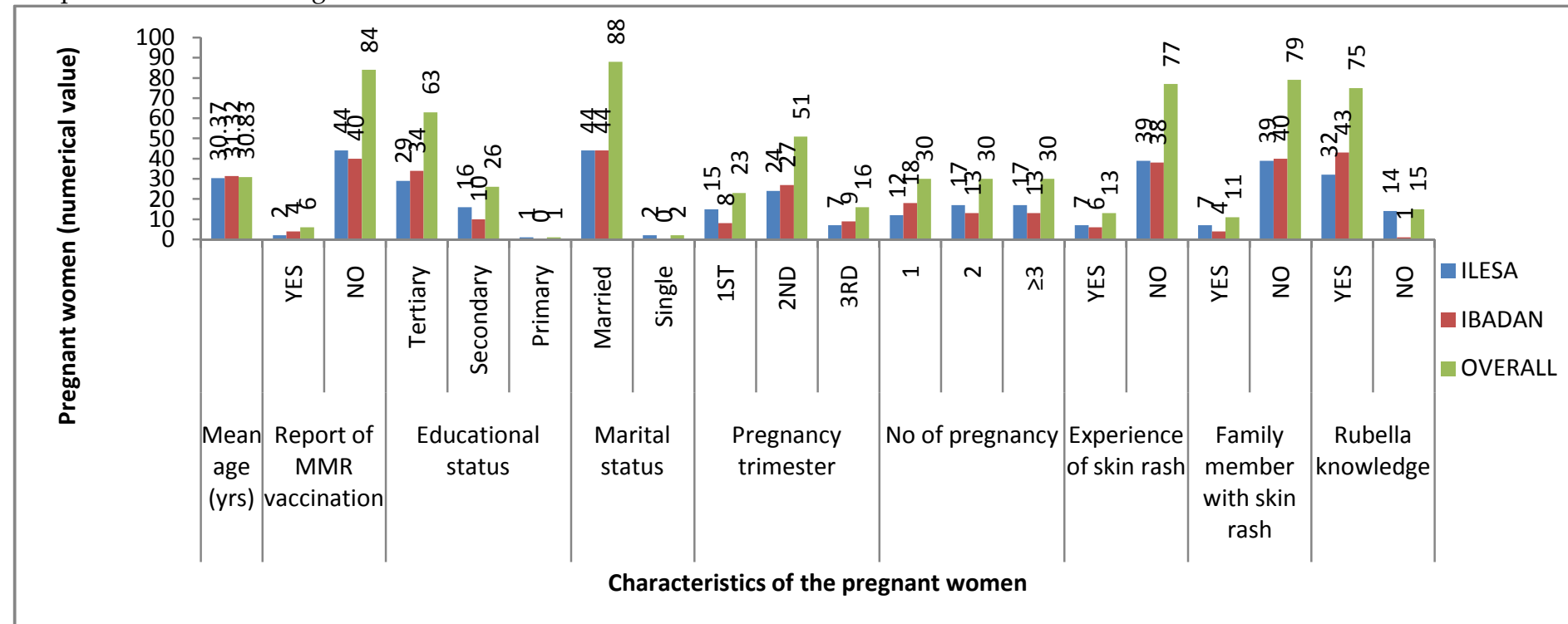

FIGURE 1: DEMOGRAPHIC CHARACTERISTICS OF PREGNANT WOMEN INVOLVED IN THE STUDY

Three pregnant women (two from Ilesa, one from Ibadan) were negative for anti-RV IgG; two of the three were negative for both anti-RV IgG and IgM antibodies.

These three were considered susceptible to RV infection, giving a rubella susceptibility rate of $3.4 \%$ $(n=89)$. The third pregnant woman (from Ilesa) $(1.1 \%$, $\mathrm{n}=90$ ) who was positive for IgM was probably in the acute phase of infection at the time of sample collection. She could therefore be a source of vertical transmission of the virus to the fetus and to other susceptible contacts.

Eighty-six $(96.6 \%, \mathrm{n}=89)$ of the pregnant women were positive for anti-RV IgG antibody while six $(6.7 \%) \quad(95 \%$ CI: $1.5-11.8, \mathrm{n}=90)$ were positive for anti-RV IgM antibody. The proportions of the pregnant women from Ilesa and Ibadan with respect to results of serologic tests are shown in Figure 2. Sera from three pregnant women (two from Ilesa and one from Ibadan) yielded equivocal IgM results. 
Comparison of anti-RV IgG and IgM prevalence rates between pregnant women from Ilesa and Ibadan revealed statistically insignificant results due to very high IgG positivity and very low IgM positivity. When the optical density (OD) of IgG was expressed in $\mathrm{WHO} \mathrm{IU} / \mathrm{ml}$ equivalent and the mean value of anti-RV IgG of the women from Ilesa was compared with that of the women from Ibadan, no significant difference was observed $(\mathrm{P}=0.06, \mathrm{n}=89)$.

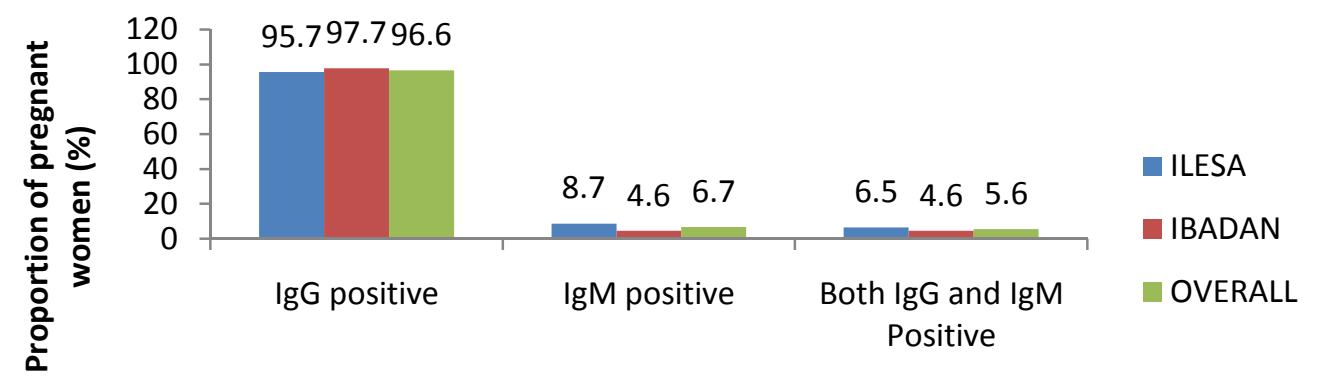

Positive serologic outcomes

\section{FIGURE 2: DETECTION OF ANTI-RUBELLA VIRUS IgG AND IgM IN PREGNANT WOMEN IN ILESA AND IBADAN}

Comparison of the mean values of anti-RV IgG concentration of the pregnant women with respect to trimester of pregnancy and parity revealed that they were statistically comparable at $P=0.62(n=89)$ and $\mathrm{P}=0.38(\mathrm{n}=89)$ respectively. Anti-RV IgG prevalence rate among pregnant women in the first and second trimester was $95.9 \%(\mathrm{n}=73)$ and corresponding IgM prevalence rate was $8.1 \%(n=74)$. Out of the 86 pregnant women positive for anti-RV IgG antibody, 6 $(7.0 \%)$ had received rubella vaccination. Comparison of the mean serum anti-RV IgG concentration of these 6 women $(28.0 \mathrm{IU} / \mathrm{ml}, 95 \% \mathrm{CI}$ : $17.11-38.87)$ with that of the remaining 80 women $(28.3 \mathrm{IU} / \mathrm{ml}, 95 \% \mathrm{CI}$ : 26.49-30.05) showed that they were statistically comparable $(\mathrm{P}=0.96)$.

\section{DISCUSSION}

In this study, we aimed at determining the proportion of pregnant women attending two tertiary hospitals in southwestern Nigeria with anti-RV IgG and IgM, the proportion susceptible to RV infection and those infectious to their unborn children. Our findings revealed that a high proportion $(96.6 \%)$ of the women at both study sites were positive for anti-RV IgG (Figure 2). While a high anti-RV IgG prevalence rate of $95.7 \%$ was detected in Ilesa where, to the best of our knowledge, no such study had been done before, the detection of $97.7 \%$ prevalence of anti-RV IgG in Ibadan is consistent with similar high prevalence of 94.2\% obtained by Adesina et al. (21) from pregnant women in Ibadan. Anti-RV IgG is valuable for all pregnant women and women of child-bearing age as it protects them and their fetuses against RV infection (22). In this study, the recorded responses of the 90 women tested showed that only six of them had received MMR vaccination which indicates that majority of those that were anti-RV IgG-positive had natural exposure to the virus and had seroconverted with protective levels of anti-RV IgG. This observation shows that Ilesa and Ibadan could be described as endemic for rubella. Comparison of mean serum IgG concentrations of women from both study sites revealed no significant difference $(\mathrm{P}=$ 0.06), a further indication of comparable endemicity of rubella in both locations.

It is noteworthy that the pregnant women seropositive for anti-RV IgG and IgM were in the first and second trimesters of pregnancy while all the 16 women in the third trimester were positive only for IgG. Anti-RV IgG and IgM prevalence rates among women in their first and second trimesters were $95.9 \%$ $(n=73)$ and $8.1 \%(n=74)$ respectively. This is contrary to the findings of Agbede et al. (23) who reported low anti-RV IgG and IgM prevalence rates of $7.0 \%$ and $1.1 \%$ respectively among 92 pregnant women in their first and second trimesters at University of Ilorin Teaching Hospital, Ilorin, Kwara State located in north-central Nigeria. This might indicate a higher susceptibility of pregnant women in their first and second trimesters of pregnancy to RV infection in north-central compared to the southwestern Nigeria. Probably, regional differences in endemicity of rubella accounted for the observed variations. Of the six pregnant women (4 from Ilesa and 2 from Ibadan) positive for anti-RV IgM, five had protective anti-RV IgG. Since these six, reportedly, did not receive rubella vaccination, it can be inferred that the five women were naturally exposed to the virus.

The prevalence rate of anti-RV IgM was low among the women in both study sites (Figure 2). This indicated low rate of rubella virus infection among the women as at the time of blood sample collection which could be due to high level of immunity to the virus (or RV endemicity) as shown by very high IgG prevalence rate. Additionally, only one of these six women reported having skin rash while two of them 
reported family members having skin rash in the past. It could not be confirmed whether the skin rash was linked to RV infection or not as five of the women had protective levels of serum IgG. Moreover, it was observed that these five pregnant women had IgG concentrations $>10 \mathrm{IU} / \mathrm{ml}$ (i.e. protective levels of anti-RV IgG) and were all in the first and second trimesters of gestation. This implies that, although they might have detectable serum anti-RV IgM concurrently with IgG, they were not possibly infectious to their fetuses or to their susceptible contacts.

Furthermore, two of the pregnant women studied were seronegative for both anti-RV IgG and IgM while another one was seropositive for only IgM. The former were considered susceptible to RV infection as they had no report of rubella vaccination and were in the first and second trimesters of pregnancy. We could, however, not state whether or not they were infected with the virus (they probably had undetectable levels of anti-RV $\operatorname{IgM}$ ) as at the time of blood sample collection. The third woman, who was negative for anti-RV IgG but positive for IgM, was a primigravida in the first trimester of gestation and had no history of rubella vaccination. This woman was most likely infected with RV as at the time of blood collection and was probably infectious to her fetus and other susceptible contacts. The fetus of this woman thus had a high risk of contracting RV from the mother with corresponding high risk of the child developing CRS (5).

Also, we observed that six pregnant women (2 from Ilesa and 4 from Ibadan) had history of rubella vaccination and were expectedly negative for anti-RV IgM with protective levels of anti-RV IgG, the mean of which was statistically comparable $(\mathrm{P}=0.96)$ to that of the remaining 80 pregnant women having protective levels of IgG. While five of these women were multiparous, only one was a primigravida. The test used in this study could however not detect whether the IgG in the sera of these women was induced by rubella vaccine or natural RV infection.

\section{REFERENCES}

1. Takahashi, S., Machikawa, F., Noda, A., Oda, T. Tachikawa, T. Detection of Immunoglobulin G and A Antibodies to Rubella Virus in Urine and Antibody Responses to Vaccine-Induced Infection. Clin Diagn Lab Immunol 1998; 5(1):24-27.

2. Hobman, T. Chantler, J. Rubella virus. In Knipe, D, M.; Howley, P. M. (eds), Fields Virology, 5th Edition, Lippincott Williams \& Wilkins, 2007; pp 1069-1070.
We also observed that overall, parity did not influence IgG concentration $(P=0.38)$ among the pregnant women.

In this study, we observed that the educational status of the pregnant women and their knowledge of rubella were generally high but the level of receipt of MMR vaccination was very low. This could be attributed to the non-inclusion of rubella vaccination in routine national immunization programme in Nigeria, probably due to endemicity of rubella in the country. It has been estimated that over 100,000 infants are born with CRS each year, mostly in developing countries that are yet to introduce rubella vaccines $(24,25)$. In this study therefore, there exists a likelihood of infecting one unborn child since the only infectious mother detected happened to be in the first trimester of gestation when up to $90 \%$ fetuses of RVinfected pregnant women may develop CRS $(26,27)$.

In conclusion, high prevalence of anti-rubella virus IgG with protective levels of the antibody was reported in pregnant women in this study. Although the rate of susceptibility to rubella as well as possibility of vertical transmission of the virus was low among the women, there was a chance of an expectant mother infecting her fetus with the virus with the possibility of RV-induced congenital abnormality. It has previously been reported by $\mathrm{WHO}$ (28) that a low level of susceptibility to RV cannot be taken to mean no risk of CRS. Therefore, based on the findings of this study, we advocate that RV immunization of all females of child-bearing age in Osun and Oyo States, and indeed throughout Nigeria, be intensified so that the likelihood of infecting their fetuses and their contacts during pregnancy can be considerably reduced.

\section{ACKNOWLEDGEMENTS}

We appreciate the permission and support of the Management and staff of Wesley Guild Hospital, Ilesa, Osun State and Jericho Nursing Home, Ibadan, Oyo State.
3. Plotkin, A.S., Reef, S.E. Rubella vaccine. In: Plotkin AS, Orenstein W, Offit P. Vaccines, 5th ed. Philadelphia, WB Saunders, 2008; pp 735-771.

4. Reef, S., Redd, S. Congenital Rubella Syndrome. In CDC's Vaccine Preventable Diseases Surveillance Manual, $4^{\text {th }}$ Edition 2008.

5. Dontigny, L., Arsenault, M-Y., Martel, M-J. Rubella in Pregnancy. J Obstet Gynaecol Can 2008; 30(2):152-158.

6. CDC. Achievements in public health: Elimination of rubella and congenital rubella syndrome - United 
States, 1969-2004. Morb Mortal Wkly Rep 2005a; 54(11):279-282.

7. CDC. Progress toward elimination of measles and prevention of congenital rubella infection, European region, 1990-2004. Morb Mortal Wkly Rep 2005b; 54:175178.

8. Robertson, S.E., Featherstone, D.A., Gacic-Dobo, M., Hersh, B.S. Rubella and congenital rubella syndrome: global update. Rev Panam Salud Publica 2003; 14:306315.

9. Gomwalk, N.E., Ahmad, A.A. Prevalence of rubella antibodies on the African continent. Rev Infect Dis 1989; 11:116-121.

10. Bhaskaram, P., Ramalakshmi, B.A., Raju, L.A., Raman, L. Need for protection against rubella in India. Indian J Pediatr 1991; 58:811-814.

11. Best, J.M., Enders, G. Laboratory diagnosis of rubella and congenital rubella. In: Banatvala JE, Peckham C (eds). Perspectives in medical virology. Vol. 15. Rubella viruses. Amsterdam, Elsevier, 2007; pp 39-78.

12. CDC. Measles, mumps, and rubella - vaccine use and strategies for elimination of measles, rubella, and congenital rubella syndrome and control of mumps: recommendations of the Advisory Committee on Immunization Practices (ACIP). Morb Mortal Wkly Rep 1998; 47(RR-8):1-57.

13. Wolinsky, J.S. Rubella virus, p. 815-840. In B. N. Fields and D. M. Knipe (ed.), Virology, 1990. Raven Press, New York, N.Y.

14. Zhang, T., Mauracher, C.A., Mitchell, L.A. Tingle, A.J. Detection of Rubella Virus-Specific Immunoglobulin G (IgG), IgM, and IgA Antibodies by Immunoblot Assays. J Clin Microbiol 1992; 30(4): 824-830.

15. Yasodhara, P., Ramalakshmi, B.A., Naidu, A.N., Raman, L. Prevalence of specific Ig $\mathrm{M}$ due to Toxoplasma, Rubella, CMV and C. trachomatis infections during pregnancy. IJMM 2001; 19 (2): 79-82.

16. Odelola, H.A., Fabiyi, A., Familusi, J.B. Distribution of rubella antibodies in Nigeria. Trans Royal Soc Trop Med Hyg 1977; 71(5):425-6.

17. Pennap, G., Amauche, G., Ajoge, H., Gabadi, S., Agwale, S., Forbi, J. Serologic Survey of Specific
Rubella Virus IgM in the Sera of Pregnant Women in Makurdi, Benue State, Nigeria. Afr J Reprod Health 2009; 13(2):69-73.

18. Bamgboye, A.E., Afolabi, K.A., Esumeh, F.I., Enweani, I.B. Prevalence of rubella antibody in pregnant women in Ibadan, Nigeria. West Afr J Med 2004; 23(3):245-8.

19. Bukbuk. D.N., el Nafaty, A.U., Obed, J.Y. Prevalence of rubella-specific IgG antibody in non-immunized pregnant women in Maiduguri, north eastern Nigeria. Cent Europ J Public Health 2002; 10(1-2):21-3.

20. Onyenekwe, C.C., Kehinde-Agbeyangi, T.A., Ofor, U.S., Arinola, O.G. Prevalence of rubella-IgG antibody in women of childbearing age in Lagos, Nigeria. $W$ Afr J Med 2000; 19(1):23-6.

21. Adesina, O.A., Adeniji, J.A., Adeoti, M.O. Rubella Ig $\mathrm{G}$ antibody in women of child-bearing age in Oyo State. Afri J Clin Exper Microbiol 2008; 9(2): 78-81.

22. Bullens, D., Smets, K., Vanhaesebrouck, P. Congenital rubella syndrome after maternal reinfection. Clin Pediatr 2000; 39:113-116.

23. Agbede, O.O., Adeyemi, O.O., Olatinwo, A.W.O., Salisu, T.J. Kolawole, O.M. Sero-Prevalence of Antenatal Rubella in UITH. The Open Public Health Journal 2011; 4:10-16.

24. Cutts, F.T., Robertson, S.E., Diaz-Ortega, J-L., Samuel, R. Control of rubella and congenital rubella syndrome (CRS) in developing countries, part 1: burden of disease from CRS. Bull WHO 1997; 75(1): 55-68.

25. Shigetaka, K. Rubella virus gene diagnosis during pregnancy and mechanism of congenital rubella. Intervirology 1998; 41: 163-169.

26. Brooks, G.F., Carroll, K.C., Butel, J.S., Morse, S.A. Paramyxoviruses and rubella virus. In: Medical Microbiology. 24th. Ed. McGraw-Hill. 2007; pp 546566.

27. De Santis, M., Cavaliere, A.F., Straface, G., Caruso, A. Rubella infection in pregnancy. Reprod Toxicol 2006; 21(4): 390-398.

28. WHO. Guidelines for surveillance of congenital rubella syndrome and rubella. Field test version; Geneva-Switzerland, 1999; p 23.5 